\title{
Permporometry study on the size distribution of active pores in porous ceramic membranes
}

\author{
G.Z. Cao*, J. Meijerink, H.W. Brinkman and A.J. Burggraaf \\ Laboratory of Inorganic Chemistry, Materials Science and Catalysis, Department of Chemical Engineering, University of \\ Twente, 7500 AE Enschede (The Netherlands)
}

(Received January 25, 1993; accepted in revised form April 21, 1993)

\begin{abstract}
Permporometry as well as nitrogen adsorption-desorption techniques have been applied to study the pore size distribution in $\gamma$-alumina membranes with a pore radius ranging from about $2 \mathrm{~nm}$ to $10 \mathrm{~nm}$. The permporometry technique measures the active pores only, while nitrogen adsorption-desorption measures the active as well as the passive pores. The pore size distribution in the porous ceramic membrane supported with an $\alpha$-alumina substrate (supported membrane) was determined by using permporometry, while the membrane without a supporting substrate (unsupported membrane) was characterized by nitrogen adsorption-desorption. The experimental results of permporometry and nitrogen adsorption-desorption indicate that the size distribution of the active pores in the supported membranes is similar to that of the active and passive pores in the unsupported membranes. The $\alpha$-alumina supporting substrate seems to have no detectable influence on the size distribution of the active pores of the ceramic membranes studied.
\end{abstract}

Key words: permporometry; pore size distribution; mesopores; porous ceramic membrane; nitrogen adsorption-desorption

\section{Introduction}

The evaluation of pore size and size distribution of the active pores in mesoporous media is important for the preparation of membranes and understanding of gas permeability/diffusion mechanisms in mesoporous media. There are a number of routine methods for measuring pore sizes and their distribution in porous media, such as gas adsorption-desorption, mercury porosimetry and thermoporometry. These methods are capable of providing information on the average pore size, pore size distribution,

\footnotetext{
To whom correspondence should be addressed.
}

porosity and specific surface area. However, they are not able to discriminate between the "active" and "passive" pores. The active pores are those effective for gas diffusion, while the passive pores are dead-end ones and do not contribute to gas diffusion through the porous media. For many applications, such as gas separation, only the active pores in porous media are important and a narrow size distribution of the active pores is essential. The gas permeability $[1,2]$ and the liquid-liquid displacement techniques [3,4] are methods for measuring the active pore size in porous media. However the gas permeability method is only able to measure the average size of the active pores. It is 
not possible to tell whether there are a large number of small pores and a small number of large pores (a broad size distribution) or all pores are of similar sizes (a narrow size distribution ). The liquid-liquid displacement method measures the size distribution of the active pores in porous media, but it is difficult to use for the characterization of small pores.

Permporometry is the only method known so far [5-12] suitable for the determination of the size distribution of the active pores with diameters ranging from about $1.5 \mathrm{~nm}$ to $0.1 \mu \mathrm{m}$ in porous media, particularly those with an asymmetric (and/or composite) structure. Permporometry, a relatively new technique, is based on the controlled blocking of the pores by capillary condensation and simultaneous measurement of the gas diffusional flux through the remaining open pores.

The present paper reports a modified permporometry set-up and a method for calculation of the size distribution of active pores in porous ceramics. The pore size distribution in porous ceramic membranes is determined by using permporometry and nitrogen adsorption-desorption. The ceramic membranes characterized by permporometry consist of two layers: a selective top layer and a supporting layer, and are referred to as supported membranes. The top layer in this study is a $\gamma$-alumina membrane and the supporting layer is a porous $\alpha$-alumina substrate with much larger pores. The $\gamma$-alumina membrane without a supporting layer is also prepared (referred to as an unsupported membrane ) and analyzed by means of nitrogen adsorption-desorption. The pore size (distribution) in supported and unsupported ceramic membranes determined by using respectively permporometry and nitrogen adsorption-desorption methods are compared and discussed.

\section{Capillary condensation}

The occurrence of capillary condensation is essential for both permporometry and nitrogen adsorption-desorption techniques. Hence the theory of capillary condensation is briefly summarized here for a better understanding of the following experiments and discussion.

When a vapour of a condensable gas is brought in contact with porous media, several mechanisms of adsorption occur successively on the inner surface of the pores as the relative pressure increases from zero to unity. With increasing relative vapour pressure, first a monomolecular layer is formed on the inner surface of the pores. As the relative vapour pressure increases further, a multi-molecular layer starts to form. Such an adsorption layer of a vapour phase on the inner surface of the pores is called "t-layer" [13,14]. It is evident that an increase in the relative vapour pressure will increase the thickness of the t-layer. Note that the t-layer forms also on a flat surface of non-porous material. When the relative vapour pressure rises further, capillary condensation will occur on the inner surface of the pores in accordance with the Kelvin equation [13,14]:

$\ln P_{\mathrm{r}}=-\frac{n \gamma_{\mathrm{s}} \nu \cos \theta}{r_{\mathrm{k}} R T}$

where $P_{\mathrm{r}}$ is the relative vapour pressure of the condensable gas, $n$ is a process parameter, $\gamma_{8}$ is the gas-solid interfacial tension $\left(\mathrm{J} / \mathrm{m}^{2}\right), \nu$ is the molar volume of the condensable gas $\left(\mathrm{m}^{3} / \mathrm{mol}\right)$, $\theta$ is the contact angle $\left(^{\circ}\right)$ and $r_{\mathrm{k}}$ the Kelvin radius $(\mathrm{m})$, which is in fact the curvature of the gas-liquid interface, $R$ is the gas constant $(\mathrm{J} /$ mol-K) and $T$ the temperature (K). As the relative vapour pressure increases further, more pores with larger Kelvin radii will be plugged. When the relative vapour pressure reaches unity, all pores will be plugged. The desorption process will occur just in the opposite way as the relative vapour pressure reduces from unity to zero. As the relative vapour pressure decreases, the liquid in large pores is removed (evaporated) first and consequently those po- 
res are opened. When the relative vapour pressure reduces further, smaller pores are also opened due to the evaporation of the liquid from those pores in accordance with the Kelvin equation. As the relative vapour pressure reduces to zero all pores are opened. It is noted that the process parameter, $n$, in the Kelvin equation (eqn. 1) is 1 for an adsorption process, while its value is equal to 2 for a desorption process $[13,14]$.

For a cylindrical pore model, the relation between the real pore radius $\left(r_{p}\right)$ and the Kelvin radius $\left(r_{k}\right)$ is as follows [15]:

$r_{\mathrm{p}}=r_{\mathrm{k}}+t$

where $t$ is the thickness of the "t-layer" formed on the inner surface of the pores. For a slitshaped pore model, the relation between the real pore width $\left(d_{\mathrm{p}}\right)$ and the Kelvin radius $\left(r_{\mathrm{k}}\right)$ is given by [15]:

$d_{\mathrm{p}}=r_{\mathrm{k}}+2 t$

\section{Experimental}

\section{Preparation of ceramic membranes}

The ceramic membrane used for the permporometry study was a La-doped $\gamma$-alumina membrane supported with a porous $\alpha$-alumina substrate (thickness about $2 \mathrm{~mm}$ ). The details of the preparation procedures were presented elsewhere [16]. The $\alpha$-alumina substrate was made from $\alpha$-alumina powder (Philips) by powder sintering and had an average pore diameter of about $0.16 \mu \mathrm{m}$ and a porosity of about $43 \%$ determined by using the mercury porosimetry method (Carlo-Erba, Series 200). The La-doped $\gamma$-alumina membrane was prepared by using the sol-gel technique and dip-coated ( 2 times) onto the $\alpha$-alumina substrate. The dip-coated membrane was then dried in a controlled moisture environment and calcined at $450^{\circ} \mathrm{C}$ for $3 \mathrm{hr}$. After the second dip-coating and calcination, the membrane was heat-treated at $1100^{\circ} \mathrm{C}$ in air for $30 \mathrm{hr}$ (referred to as type $\mathrm{A}$ membrane). With similar procedures, another specimen was made but this time, the membrane was heat-treated at $1000^{\circ} \mathrm{C}$ in air for 30 hr (referred as type B membrane). The thickness of the La-doped $\gamma$-alumina membrane (for both type A and type B membranes) is about 5 $\mu \mathrm{m}$ according to SEM observation. Moreover, for comparison with literature data $[10,11]$, a $\gamma$-alumina membrane (supported with $\alpha$-alumina but without La-dopant), the same type as used in Refs. [10,11], was prepared using the sol-gel technique and subjected to permporometry study. This $\gamma$-alumina (referred as type $\mathrm{C}$ membrane) was only calcined at $450^{\circ} \mathrm{C}$ for 3 $\mathrm{hr}$ and no further heat-treatment was applied.

For the nitrogen adsorption-desorption analysis, unsupported La-doped $\gamma$-alumina membranes were also prepared from the same sol (colloidal solution) with the respective heattreatment for the supported type $A$ and type $B$ membranes. The details of the preparation of the unsupported La-doped $\gamma$-alumina membranes were presented elsewhere [17].

\section{Permporometry}

A schematic diagram of the experimental setup of permporometry is given in Fig. 1. This homemade apparatus consists of four parts. The first part is the gas flow system, including the condensable vapour generators, spiral coolers, mass flow controllers, a differential pressure transducer and gas mixers. The second part is the diffusion cell, where the capillary condensation inside pores and the gas diffusion through the remaining open pores take place. The third part is the gas chromatograph and a data recorder. The fourth part is the temperature-controlling system, including the water bath etc.

A mixture of cyclohexane vapour, acting as a condensable gas, and nitrogen gas was flushed 


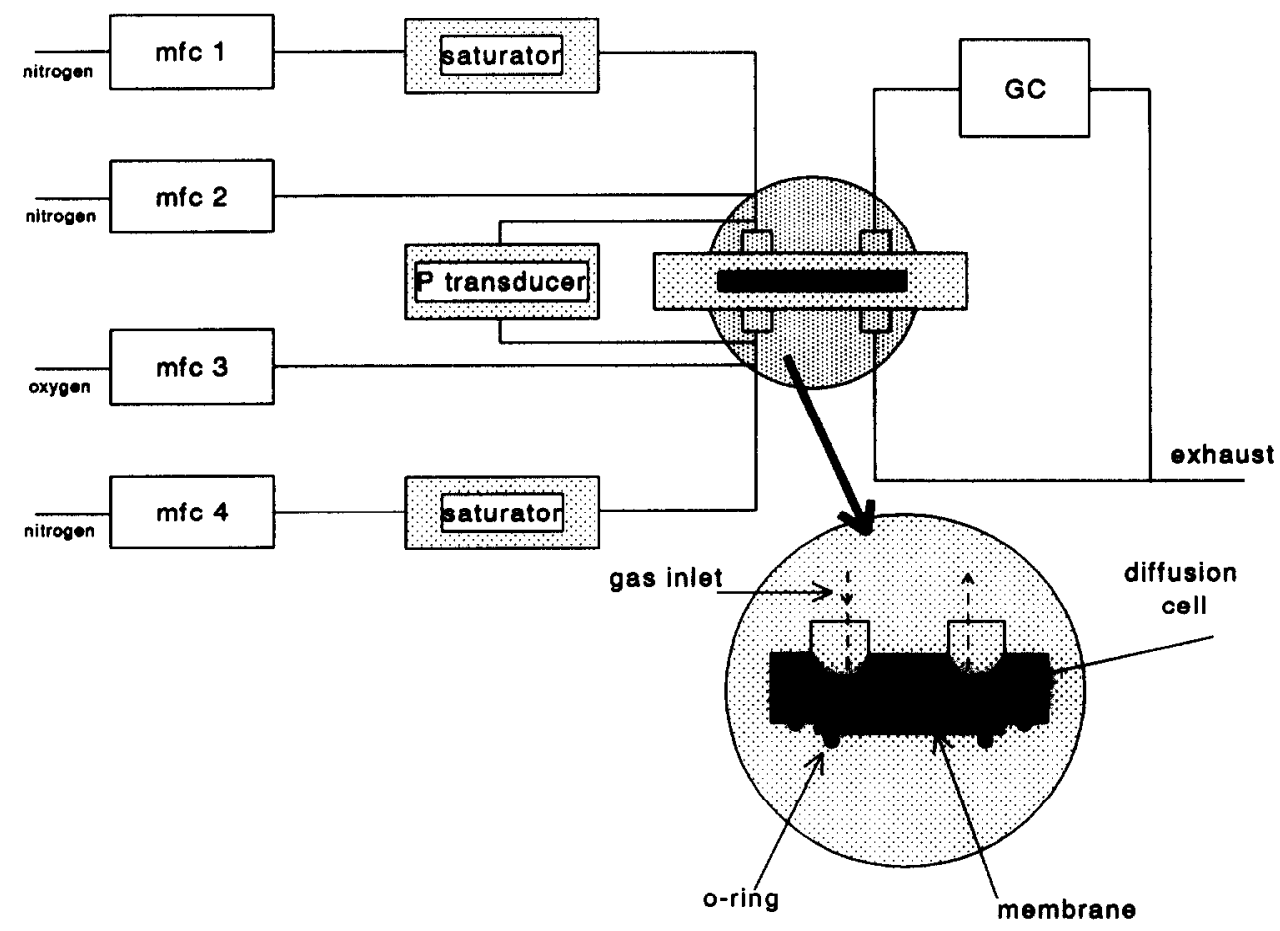

Fig. 1. A schematic diagram of the permporometry set-up.

at the membrane side of the specimen. The other side (supporting layer) of the specimen was flushed with a mixture of cyclohexane vapour, nitrogen and oxygen gases. In theory, the orientation in which the samples are placed in the diffusion cell should not influence the experimental results. The total pressure inside the diffusion cell was about 1 bar and the total gas flux along each side of the specimen was about $50 \mathrm{ml} / \mathrm{min}$. The condensable gas can be any vapour, provided that it has a reasonable vapour pressure, a high evaporation rate and is inert to the specimens to be characterized. Besides, the condensable gas should also have a good wettability and consist of small, spheric molecules. $\mathrm{CCl}_{4}$ should be an ideal candidate [9] but is also a very poisonous gas, and thus is not used in our experiments. According to literature $[10,11]$ cyclohexane is a good candidate and hence was chosen for our experiments. Nitrogen was used as a carrier gas for the condensa- ble gas while oxygen gas was added for the determination of the gas diffusion through the membranes; the oxygen content was measured by using the gas chromatograph. From the permporometry set-up one can see that the oxygen partial pressure $\left(P_{\mathrm{O}_{2}}\right.$, on the high oxygen partial pressure side) varies with $1-P_{\mathrm{r}}, P_{\mathrm{r}}$ being the relative vapour pressure of cyclohexane. The gas diffusion through the pores of porous ceramic membranes in the present study is in the region of Knudsen diffusion (see Appendix). Since there is no overall pressure gradient across the membranes, the oxygen gas transport through the substrates is due to the oxygen gas concentration gradient (or the oxygen differential partial pressure) across the specimens. Thus the oxygen gas transported through the substrates is assumed to be proportional to the oxygen differential partial pressure across the substrates in the pressure range measured (from 0 to $1 \mathrm{~atm}$ ). The oxygen 
diffusional flux through the specimens was corrected for the driving force (the oxygen differential partial pressure), which hereafter is referred to as oxygen permeability. Note that the oxygen permeability presented in this paper is in fact the oxygen permeability through the specimens consisting of both the top-layer membrane and the supporting layer. The permporometry experiments reported in the present paper were all conducted using the desorption process. This is because a shorter time is needed to get an equilibrium condition according to our experience. The equilibrium time was about $20-40 \mathrm{~min}$ for desorption measurements and much longer for adsorption ones. The temperature of the diffusion cell was kept at $15^{\circ} \mathrm{C}$.

At the start of the measurements both sides of the membrane in the diffusion cell were flushed with the (mixed) gases saturated with cyclohexane vapour. All pores in the membrane were then filled with liquid cyclohexane due to the capillary condensation and no gas diffusion through the membrane was possible. When the vapour pressure was subsequently reduced, pores with Kelvin radii larger than a corresponding size (determined by the Kelvin equation) were opened and available for gas diffusion. By stepwise decreasing the vapour pressure, the oxygen diffusion flow through the membrane was measured, from which the size distribution of the active pores was calculated graphically (for details see later in the Results section) by using the following equation (see Appendix ):

$f(r)=-\frac{3 \tau l}{2 r^{3}} \sqrt{\frac{M R T}{8 \pi}} \frac{\mathrm{d} F}{\mathrm{~d} r}$

where $f(r)$ is the size distribution of the active pores $\left(1 / \mathrm{m}^{3}\right.$, the number of active pores with radius from $r$ to $r+d r$ per unit volume) and $F$ is the oxygen permeability $\left(\mathrm{mol} / \mathrm{m}^{2}\right.$-sec-Pa). The details of the derivation of this equation and the definition of the other symbols are given in the Appendix. Note that the calculation of the size distribution of the active pores was performed using a cylindrical pore model. This will be discussed later in the discussion section.

The pore size distribution obtained by using permporometry is in fact the size distribution of the Kelvin radii of the active pores. As discussed previously the real pore radius is the Kelvin radius plus the thickness of the t-layer for a cylindrical pore model, $r_{\mathrm{k}}+t$, (eqn. 2), while for a slit-shaped pore model the real pore width is equal to $r_{k}+2 t$ (eqn. 3 ). The thickness of the t-layer could be determined by separate adsorption experiments using homogeneous non-porous reference surfaces. However, it would be very difficult to prepare reference surfaces from the same material having the same surface chemical properties, and the measurement is very laborious. In the present study we used the method proposed by Cuperus et al. $[10,11]$ to estimate the thickness of the t-layer directly from permporometry data. The procedure is outlined in the Results section.

\section{Nitrogen adsorption-desorption}

The nitrogen adsorption-desorption technique was also used for determining the pore size distribution of unsupported La-doped $\gamma$-alumina membranes. The measurement experiments were conducted using a Micromeritics ASAP 2400 (at liquid nitrogen temperature, 77 K). Since we have used the desorption process in the permporometry experiment, we also use the nitrogen desorption isotherm to calculate the pore size distribution and the average pore size (using a cylindrical pore model for the type $A$ and $B$ membranes and a slit-shaped pore model for the type $\mathrm{C}$ membrane, to be discussed later).

\section{Results}

Figure 2 shows the cumulative oxygen 
permeability as a function of the relative vapour pressure of cyclohexane for the type A Ladoped alumina membrane (supported membrane). The cumulative oxygen permeability curve can be divided into three parts. When the relative vapour pressure is above 0.8 , all pores are plugged by liquid cyclohexane, and there is no substantial oxygen diffusion through the membrane. As the relative vapour pressure decreases from 0.8 to 0.7 (the relative oxygen partial pressure at the oxygen feed side is about $0.2-0.3$ ), the cumulative oxygen permeability increases drastically. This increase of oxygen permeability is evidently due to the desorption of cyclohexane from the pores, so that the pores are open for oxygen gas diffusion through the membrane. A further decrease in the relative vapour pressure of cyclohexane results only in a slight increase of oxygen permeability. Here all pores are open, nevertheless there is a very small slope. This is due to the change of the thickness of t-layer on the inner surface of the pores. The thickness of the $t$-layer decreases with the relative vapour pressure, and conse- quently the effective pore size for gas diffusion increases, and thus the oxygen permeability increases slightly. The permporometry measurements have been repeated and identical experimental results were obtained by using either the same specimen or another specimen from the same batch. It is clear that the permporometry measurements show a very good reproducibility and that the porous ceramic membranes (from the same batch) used in the present study have a consistent pore size distribution.

The relations between the cumulative oxygen permeability and the relative vapour pressure of cyclohexane in Fig. 2 can be converted to relations between the cumulative oxygen permeability and the Kelvin radii as shown in Fig. 3, in accordance with the Kelvin equation (eqn. A-2, details see in Appendix). In Fig. 3 the cumulative oxygen permeability is plotted as a function of the Kelvin radius and from this curve the size distribution of the Kelvin radii of active pores in the type A membrane was calculated using eqn. (4). The size distribution of

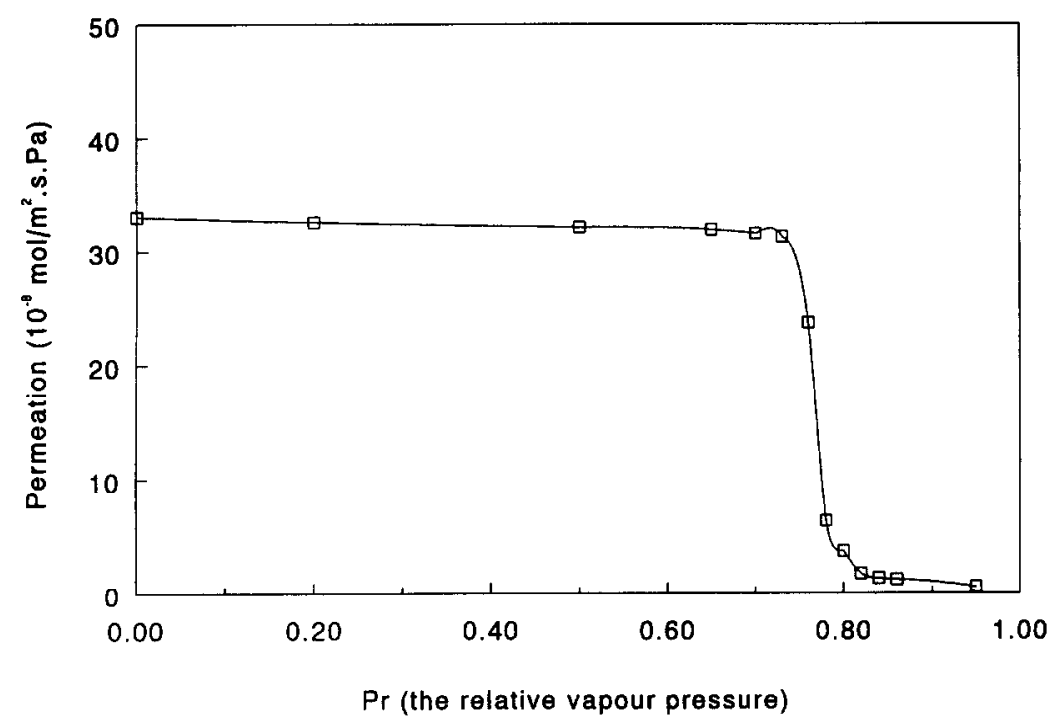

Fig. 2. Oxygen permeability as a function of the relative vapour pressure of cyclohexane, during desorption process. 


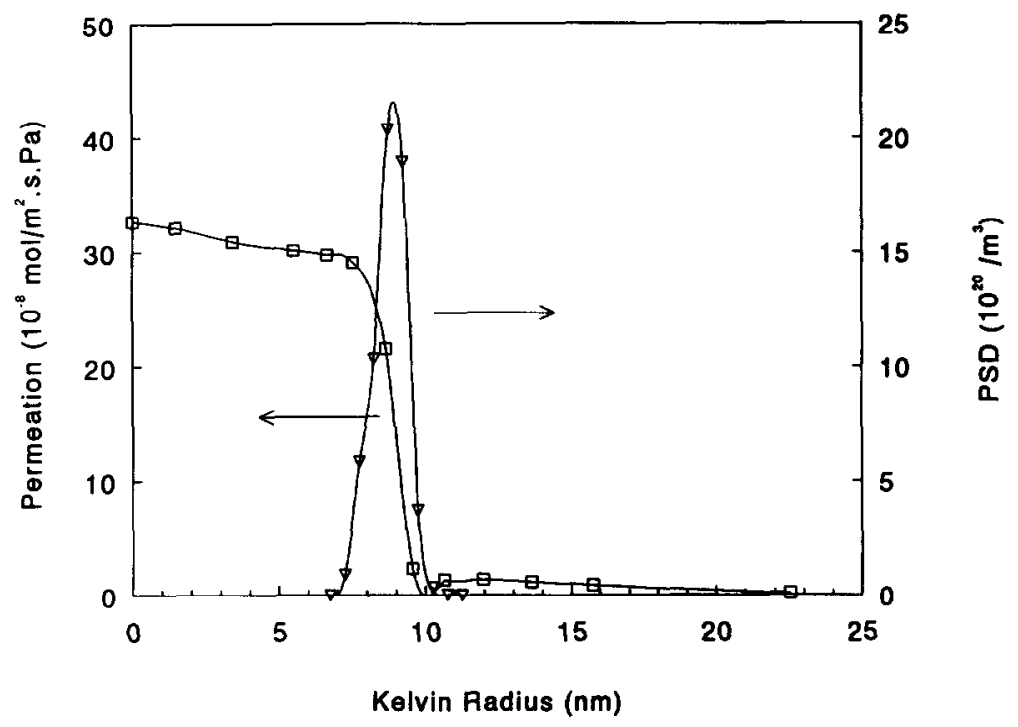

Fig. 3. Oxygen permeability as a function of Kelvin radius and the pore size distribution (PSD) for the type A supported membrane determined by permporometry.

the Kelvin radii of the active pores is also presented in Fig. 3. For calculating the pore size distribution, the interval of Kelvin radii, $d r$, was chosen to be $0.5 \mathrm{~nm}$ and accordingly the interval of cumulative oxygen permeability, $\mathrm{d} F$, was taken graphically from the curve. The tortuosity ( $\tau$, the square of the ratio of the real pore length to the thickness of the specimen) in the La-doped $\gamma$-alumina membrane is assumed to be 3 [18] and the diffusion resistance of the supporting $\alpha$-alumina substrate is assumed to be $70 \%$ of the total flow resistance according to the literature [19]. These assumptions are also applied in the following calculations unless otherwise specified. The assumptions will affect the data of $f(r)$ in eqn. (4) (number of pores), but the size distribution of active pores in the membranes will not be changed and consequently the average pore size of the active pores in the membranes will be the same. The number averaged pore size is calculated from the pore size distribution using the following equation:

$$
\bar{r}=\frac{\int_{0}^{\infty} r f(r) \mathrm{d} r}{\int_{0}^{\infty} f(r) \mathrm{d} r}
$$

Figure 3 clearly shows a sharp pore size distribution with an average Kelvin radius $\left(r_{k}\right)$ of 9 $\mathrm{nm}$. The long tail of the cumulative oxygen permeability at $r_{k}>10 \mathrm{~nm}$ in Fig. 3 (or $P_{\mathrm{r}}>0.8$ in Fig. 2) is probably an indication of a small amount of large (larger than $10 \mathrm{~nm}$ ) pores also existing in the specimen.

The thickness of the t-layer is then estimated directly from the average Kelvin radius obtained above and the oxygen diffusion data by using the method proposed by Cuperus et al. $[10,11]$. As discussed above (see Fig. 2), when the relative vapour pressure is reduced to 0.7 , all pores (in the type A supported membrane) are open for oxygen diffusion. Under such a condition, the effective pore radius for oxygen diffusion is the Kelvin radius. Thus according 
to eqn. (4) the oxygen permeability $F_{0.7}$ measured is proportional to the cube of the average Kelvin radius, $r_{\mathrm{k}}$. When the relative vapour pressure is subsequently reduced to zero, there is no t-layer present on the inner surface of the pores and so the oxygen permeability $F_{0}$ measured is proportional to the third power of the (real) average pore radius $r_{\mathrm{p}}\left(=r_{\mathrm{k}}+t\right)$, assuming a cylindrical pore model. Combining the oxygen permeability at $P_{\mathrm{r}}=0$ and 0.7 , we have the following relation:

$\frac{F_{0.7}}{r_{\mathrm{k}}^{3}}=\frac{F_{0}}{\left(r_{\mathrm{k}}+t\right)^{3}}$

Hence the thickness of the t-layer (at $P_{\mathrm{r}}=0.7$ ) can be calculated and is found to be approximately $0.5 \mathrm{~nm}$, which agrees well with that reported by Cuperus et al. [10,11]. This thickness is used as the average value for all pores, ignoring the change of the thickness of the $t$ layer as the relative vapour pressure of cyclohexane varies from 0.7 to 0.8 . Taking the t-layer thickness of $0.5 \mathrm{~nm}$ into account (using eqn. $2)$, the average pore radius $\left(r_{p}\right)$ of the active pores in the type A supported La-doped $\gamma$-alumina membrane is $9.5 \mathrm{~nm}$.

The La-doped $\gamma$-alumina membrane without $\alpha$-alumina support (the type A unsupported membrane) but prepared with the same heattreatment as for the type A supported membrane was analyzed by means of the nitrogen adsorption-desorption technique. Its pore size distribution is presented in Fig. 4, which is obtained from the nitrogen desorption isotherm. The average pore size obtained is about $10 \mathrm{~nm}$ in radius (including the thickness of t-layer), which agrees well with that $(9.5 \mathrm{~nm})$ obtained from the permporometry measurement. However, it is seen that the pore size distribution in the supported membrane determined by permporometry, is sharper than that in the unsupported one, determined by nitrogen adsorption-desorption. One should realize that the pore size (distribution) measured by using the nitrogen adsorption-desorption technique is the size (distribution) of the active as well as passive pores. On the other hand, permporometry measures only the active pores.

Permporometry study was also performed on the type $B$ supported membrane. Figure 5 shows the cumulative oxygen permeability as a function of the Kelvin radius, and the size distribution of the Kelvin radii of active pores for the type B supported membrane. The average Kelvin radius calculated from this size distribution curve is about $4.8 \mathrm{~nm}$. The thickness of the tlayer is estimated to be about $0.5 \mathrm{~nm}$. Thus the average active pore size in the type $B$ supported membrane is approximately $5.3 \mathrm{~nm}$ in radius using a cylindrical pore model. Furthermore the nitrogen adsorption-desorption analysis was also conducted on the La-doped $\gamma$-alumina membrane (the type $B$ unsupported membrane ) without the $\alpha$-alumina supporting layer but otherwise prepared in the same way as the type $\mathrm{B}$ membranes. The pore size distribution is shown in Figure 6 and the average pore size is calculated to be about $5.5 \mathrm{~nm}$ in radius, very close to that $(5.3 \mathrm{~nm})$ of the active pores obtained from the permporometry measurement. It is seen that the mean pore size (of active and passive pores) in the type B (supported and unsupported) membranes is much smaller than in type A membranes as expected from their different heat-treatments. For both type A and $B$ membranes, the pore size in unsupported membranes, determined by using nitrogen adsorption-desorption, is somewhat larger than in supported ones determined by permporometry.

Figure 7 gives the cumulative oxygen permeability as a function of the Kelvin radii and the size distribution of the Kelvin radii of active pores in the type $\mathrm{C}$ membrane. The average Kelvin radius is about $2.2 \mathrm{~nm}$, which is larger than $1.5 \mathrm{~nm}$, the average Kelvin radius in $\gamma$ alumina membranes reported in literature $[10,11]$. According to literature [20] a slit- 


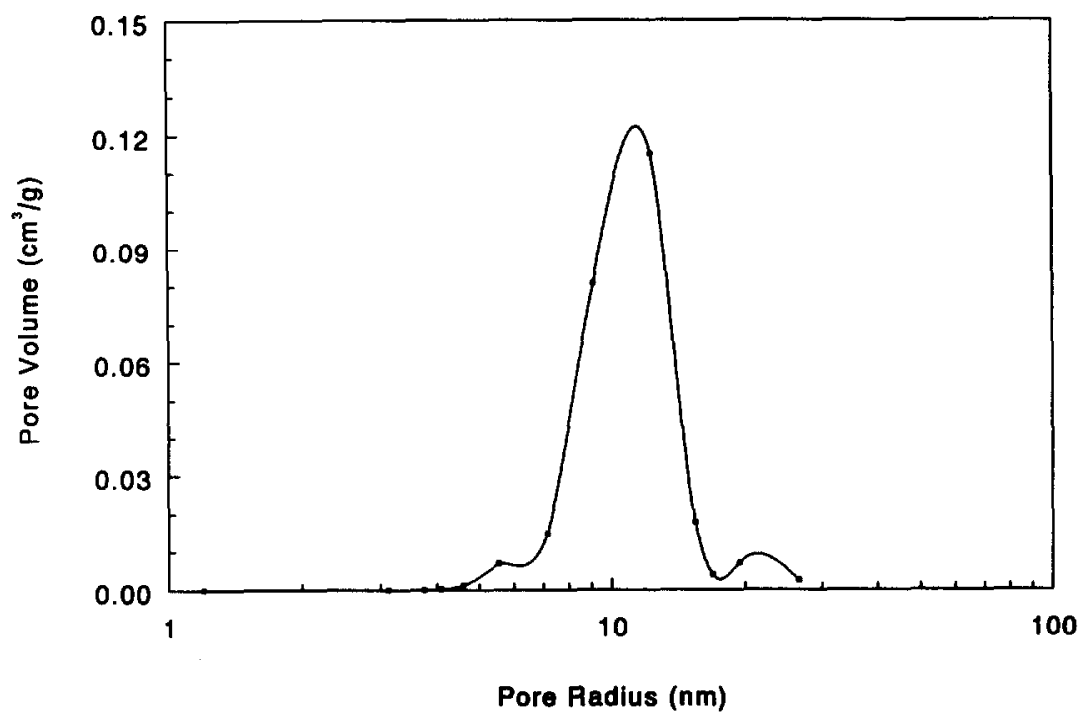

Fig. 4. The pore size distribution (PSD) in the type A unsupported membrane, determined by the nitrogen adsorptiondesorption technique.

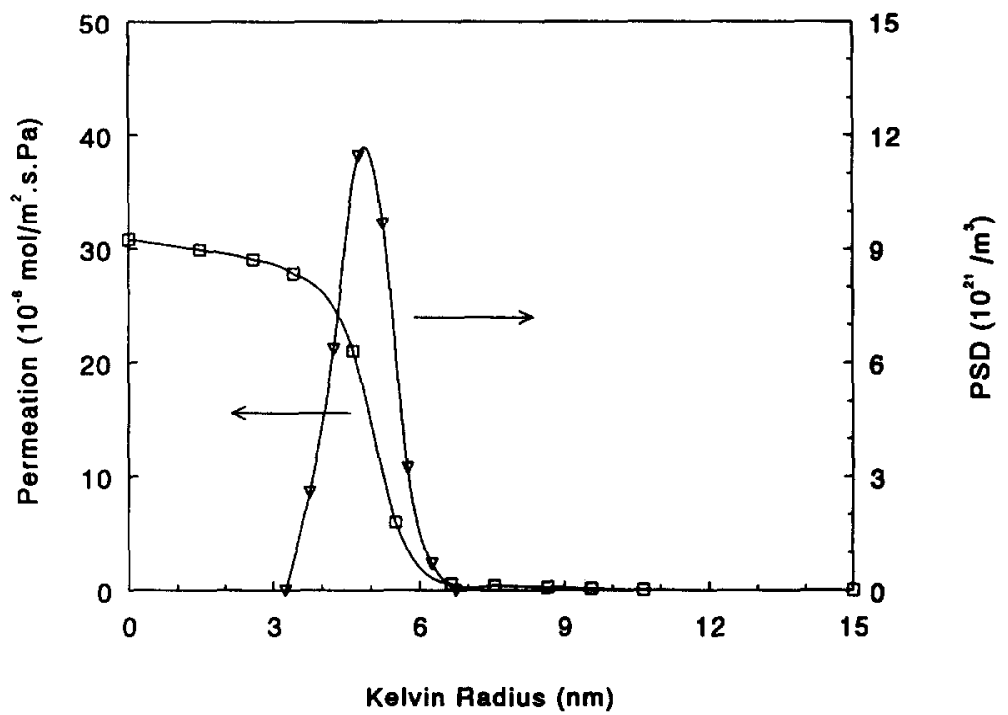

Fig. 5. Oxygen permeability as a function of Kelvin radius and the pore size distribution (PSD) for the type B supported membrane determined by permporometry.

shaped pore model should be used for this membrane, the average width of active pores is then calculated to be approximately $3.2 \mathrm{~nm}$ using eqn. (3). This result agrees well with the data $\left(d_{\mathrm{p}}=3 \mathrm{~nm}\right)$ obtained using nitrogen ad- sorption-desorption method [20,21].

Also the bulk porosity of the three (type A, $B$ and C) different unsupported $\gamma$-alumina membranes were measured by means of nitrogen adsorption-desorption and were found to 


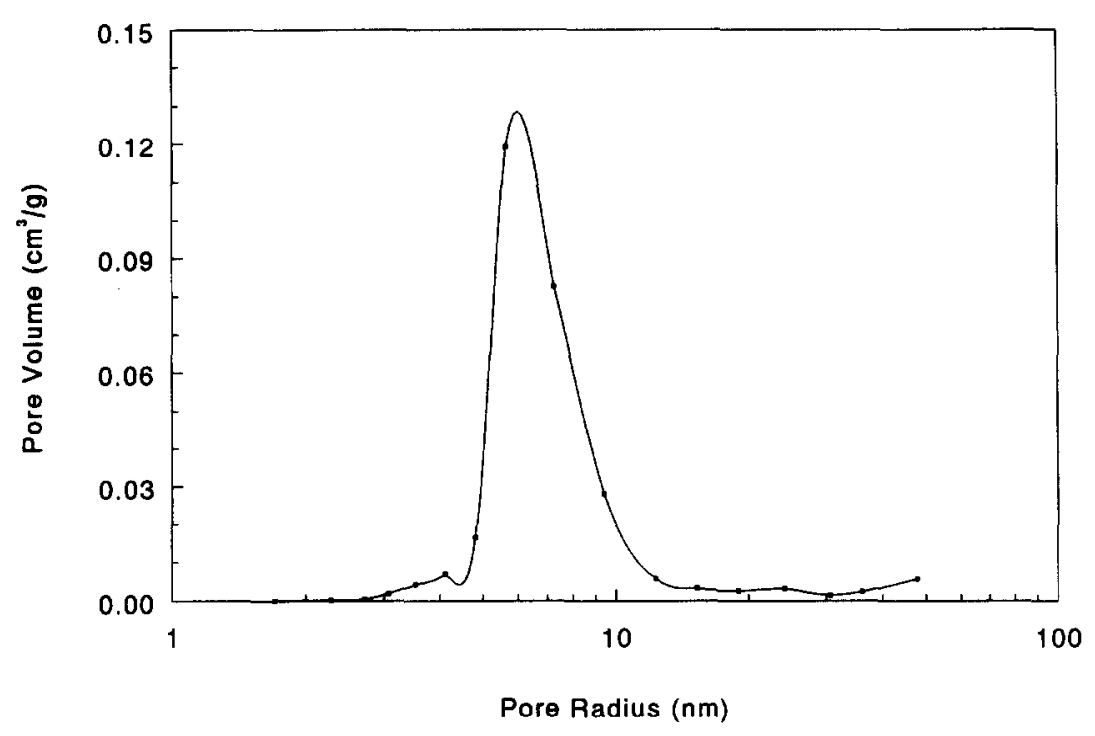

Fig. 6. The pore size distribution (PSD) in the type B unsupported membrane, determined by the nitrogen adsorptiondesorption technique.

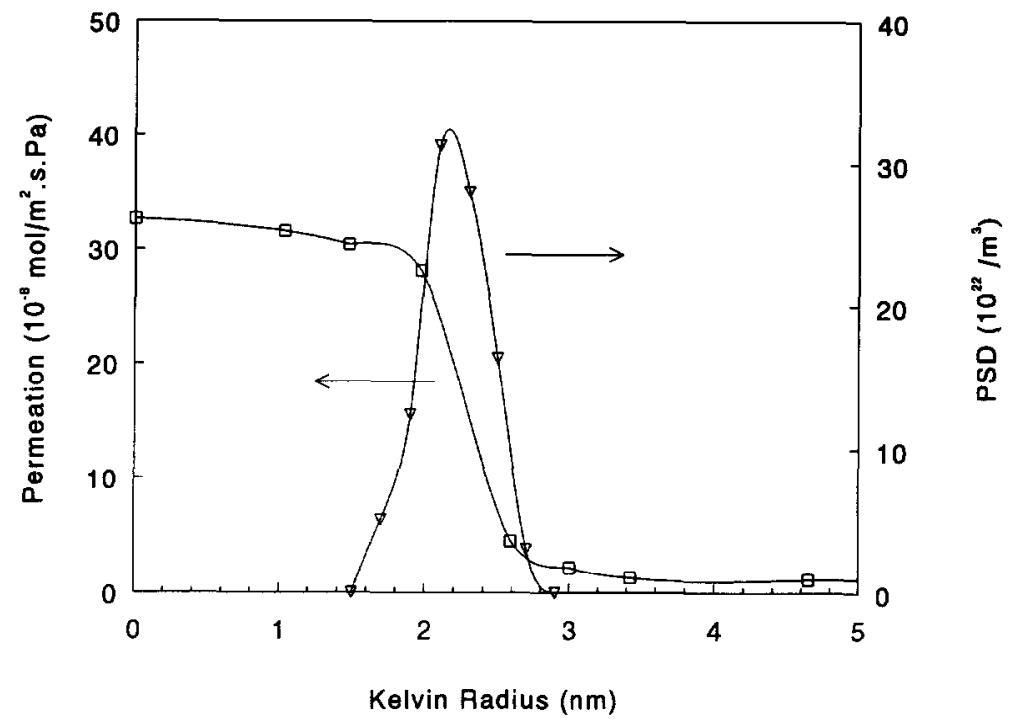

Fig. 7. Oxygen permeability as a function of Kelvin radius and the pore size distribution (PSD) for the type C supported membrane determined by permporometry.

be the same, i.e. approximately $50 \%$. Obviously during the further heat-treatment, small pores in the membranes merge into large ones, but no pore volume is eliminated from the membranes and the porosity remains the same. 


\section{Discussion}

Both permporometry and nitrogen adsorption-desorption techniques are based on capillary condensation inside the pores of porous media. Thus they are capable of determining the pore size distribution in porous media with pore radii ranging from about $1.5 \mathrm{~nm}$ to $0.1 \mu \mathrm{m}$. However there is also an essential difference between these two methods. In permporometry experiments capillary condensation is applied for controlled blocking of the pores and simultaneously the gas permeability through the remaining pores is measured. The pore size (distribution) is then calculated from the gas diffusion data. Thus only those pores effective (active pores) for gas diffusion are taken into account. The dead-end pores, which have no contribution to the gas diffusion, are not measured. Furthermore in permporometry studies only the radius of the narrowest part of each pore is measured, if a pore has no uniform size along the longitudinal direction. It is obvious that the gas diffusion through a pore is possible only when the pore is open from one side to the other. In permporometry experiments, a pore with a uniform size is opened at once when the relative vapour pressure of cyclohexane is reduced below a certain level in accordance with the Kelvin equation, and is thus available for gas diffusion. However for a pore having no uniform size along its longitudinal direction, the part with large size (radius) will be opened first as the relative vapour pressure decreases in a desorption process, but the pore will not be available for gas diffusion until the relative vapour pressure reduces to such a level that the smallest part of the pore is also opened. The same principle holds if an adsorption process is used, in which the narrowest part of a pore is filled first. Therefore the pore size (distribution) obtained by using permporometry is always the narrowest part of each pore in porous media. In the nitrogen adsorption-desorption study the capillary condensation of nitrogen inside the (active as well as passive) pores is measured and the data are directly used for the calculation of the pore size distribution. Both methods measure the smallest (interconnecting) pathways of the active pores in porous materials (desorption branch is used). Obviously the smallest effective pathways (i.e. the active pore size) have the same dimension for active as well as passive pores in the membranes studies.

In the present study the calculation of the pore size distribution of both supported and unsupported La-doped $\gamma$-alumina membranes was performed using a cylindrical pore model (eqn. 4, details see in Appendix). It is clear that only when porous media have ideal cylindrical pores with very well determined tortuosity and flow resistance in the supporting layer, the pore size distribution $f(r)$ can be treated quantitatively. However, in practice, in porous media irregular pore geometry, such as conical, slitshaped and mixed shaped, is quite common. Besides, the tortuosity and the flow resistance in the supporting layer are very difficult to measure precisely. Therefore the pore size distribution, namely $f(r)$, in porous media determined by using permporometry is an effective distribution of the smallest pathways and should be treated only semi-quantitatively.

According to literature $[20,21], \gamma$-alumina membranes, prepared by using the sol-gel technique and calcined at $450-600^{\circ} \mathrm{C}$ in air for $3 \mathrm{hr}$, have plate-like particles and thus the pores (both active and passive) are considered to be slit-shaped. However, both supported and unsupported La-doped $\gamma$-alumina membranes used (both type A and B) in the present study, have been subjected to a further heat-treatment at 1000 or $1100^{\circ} \mathrm{C}$ in air for $30 \mathrm{hr}$. After such a heat-treatment the pores in the membranes have grown from about $2-3 \mathrm{~nm}$ in diameter to about $10-20 \mathrm{~nm}$. The membranes after such a heat-treatment have a nitrogen ad- 


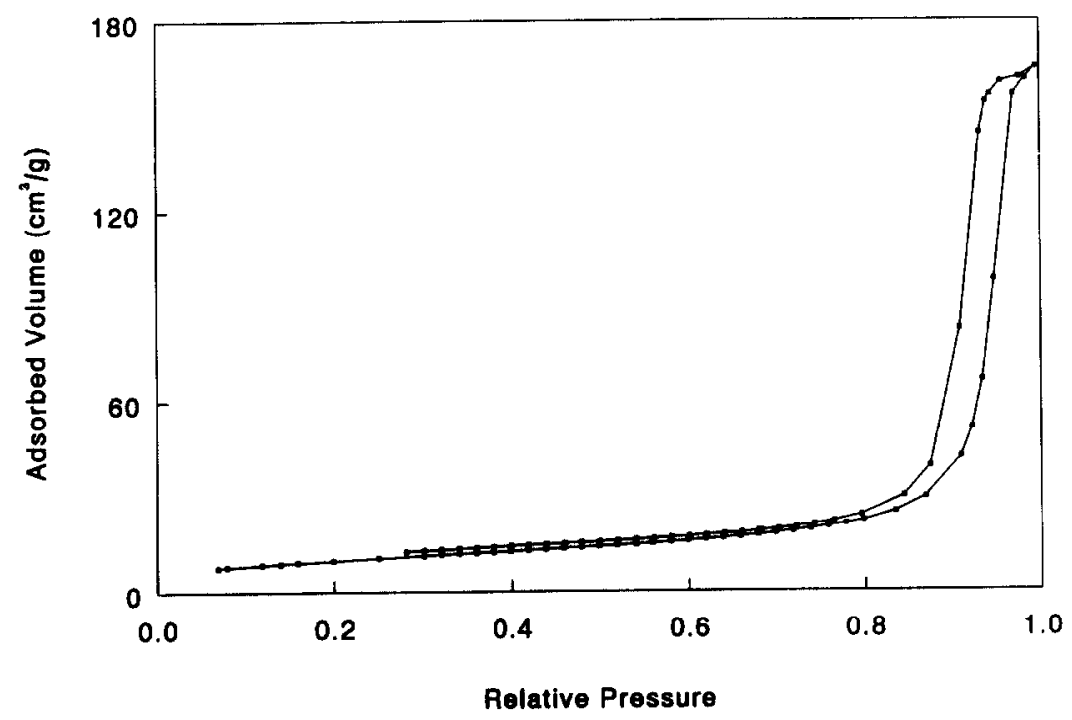

Fig. 8. Nitrogen adsorption-desorption isothermal at $77 \mathrm{~K}$ for the type A unsupported membrane.

sorption-desorption isothermal as shown in Fig. 8, which is a typical isothermal for porous materials having cylindrical or cylinder-like (including conical, ink-bottle, etc.) pores [22,23].

From Figs. 3, 5 and 7 it can be seen that the average pore size in the membranes studied increases with the heat-treatment temperatures, while the number of pores, $f(r)$, decreases accordingly. Furthermore, the oxygen permeabilities through the (three types of) membranes studied were found to be very close to each other at $P_{\mathrm{r}}=0$ (i.e. no capillary condensation occurs and all pores are open). This result implies that during the heat-treatment, small pores merge to form large pores, which results in an increase of pore size and a decrease of the number of pores. However the heat-treatment has no evident influence on the oxygen permeability through the membranes. More detailed study on the evolution of the membrane microstructure during the heat-treatment is beyond the scope of this paper. It can also be seen that the size distribution of active pores in supported membranes becomes broader when a higher temperature is used for the heat-treatment. In the type $\mathrm{C}$ membrane (no further heat-treatment) the broadness of the pore size distribution is less than $1.5 \mathrm{~nm}$, and it is about $3 \mathrm{~nm}$ for the type $\mathrm{B}$ membrane (heat-treated at $1000^{\circ} \mathrm{C}$ for $30 \mathrm{hr}$ ), and $4 \mathrm{~nm}$ for the type A membrane (at $1100^{\circ} \mathrm{C}$ for $30 \mathrm{hr}$ ). The reason for this is not yet clear.

\section{Conclusions}

This paper reports a modified permporometry set-up and a method for calculation of the size distribution of active pores in porous media. The present work shows clearly that permporometry is a unique technique for determining the pore size and size distribution of active pores in mesoporous media, especially for those with an asymmetric structure (supported membranes) and pore radius ranging from about $1.5 \mathrm{~nm}$ to $0.1 \mu \mathrm{m}$.

Experimental results from both permporometry and nitrogen adsorption-desorption studies indicate that the La-doped $\gamma$-alumina membranes heat-treated at $1000-1100^{\circ} \mathrm{C}$ for 30 
hr have an average pore radius of about 5.5 to $10 \mathrm{~nm}$, and have a cylindrical pore shape. $\gamma-\mathrm{Al}-$ umina membranes [without La-dopant and heat-treated at a relatively low temperature $\left.\left(450^{\circ} \mathrm{C}\right)\right]$ have slit-shaped pores with an average pore width of about $3.2 \mathrm{~nm}$.

\section{Acknowledgements}

This investigation was supported by the Dutch Ministry of Economic Affairs (Research Project 'ECVD Synthesis and Properties of Porous Composite Systems'. IOP Technical Ceramics No. 87.A045). The authors would like to acknowledge Ing. J. Hekkink for building the permporometry set-up, and Dr. K.J. de Vries, Dr. K. Keizer and Dr. G.W. Koebrugge for fruitful discussion.

\section{List of symbols}

$P_{\mathbf{r}} \quad$ relative vapour pressure of cyclohexane (-)

$P_{\mathrm{O}_{2}} \quad$ oxygen partial pressure (-)

$\theta$ contact angle $\left({ }^{\circ}\right)$

$\gamma_{\mathrm{s}}$ interfacial tension $\left(\mathrm{J} / \mathrm{m}^{2}\right)$

v molar volume $\left(\mathrm{m}^{3} / \mathrm{mol}\right)$

$r_{\mathbf{k}} \quad$ Kelvin radius describing the curvature of the liquid-gas interface (m)

$r, \rho$ pore radius $(\mathrm{m})$

$r_{\mathrm{p}} \quad$ pore radius for cylindrical pores $(\mathrm{m})$

$d_{\mathrm{p}} \quad$ pore width for slit-shaped pores $(\mathrm{m})$

$l$ thickness of the (top-layer) porous membranes (m)

$F \quad$ gas permeability $\left(\mathrm{mol} / \mathrm{m}^{2}-\mathrm{sec}-\mathrm{Pa}\right)$

$M \quad$ molar mass of the gas $(\mathrm{kg} / \mathrm{mol})$

$\tau \quad$ tortuosity (-)

$t \quad$ thickness of $t$-layer $(m)$

$f(r)$ pore size distribution $\left(1 / \mathrm{m}^{3}\right)$

$\epsilon \quad$ surface porosity (-)

$v$ the average molecular velocity $(\mathrm{m} / \mathrm{sec})$

$N$ the number of pores per unit surface area $\left(1 / \mathrm{m}^{2}\right)$ $n$ the process parameter in adsorption-desorption (-)

$R \quad$ gas constant (J/mol-K)

$T$ temperature (K)

$\alpha, \gamma$ greek letters to describe the alumina phases

\section{References}

1 H. Yasuda and J.T. Tsai, Pore size of microporous polymer membranes, J. Appl. Polym. Sci., 18 (1974) 805-819.

2 R.J. van Vuren, B.C. Bonekamp, K. Keizer, R.J.R. Uhlhorn, H.J. Veringa and A.J. Burggraaf, Formation of ceramic alumina membranes for gas separation, in: P. Vincenzini (Ed.), High Tech Ceramics, Elsevier, Amsterdam, 1987, pp. 2235-45.

3 H. Bechhold, M. Schlesinger and K. Silbereisen, Porenweite von ultrafiltern, Kolloid Z., 55 (1931) 172.

4 S. Munari, A. Bottino, P. Moretti, G. Capanelli and I. Becchi, Permporometric study on ultrafiltration membranes, J. Membrane Sci., 41 (1989) 69-86.

5 C. Eyraud, M. Betemps, J.F. Quinson, F. Chatelut, M. Brun and B. Rasneur, Détermination de la répartition des rayons de pores d'un ultrafiltre, Bull. Soc. Chim. France, 9-10 (1984) I-237-244.

6 A. Mey-Marom and M.G. Katz, Measurement of active pore size distribution of microporous membranes. A new approach, J. Membrane Sci., 27 (1986) 119130 .

7 M.G. Katz and G. Baruch, New insights into the structure of microporous membranes obtained using a new pore size evaluation method, Desalination, 58 (1986) 199-211.

8 C. Eyraud, Application of gas-liquid permporometry to characterization of inorganic ultrafilters, in: $\mathbf{E}$. Drioli and M. Nakagaki (Eds.), Membranes and Membrane processes, Plenum Press, New York, NY, 1986, pp. 629-634.

9 D.E. Fain, A dynamic flow-weighed pore size distribution, in: J. Charpin and L. Cot (Eds.), First Int. Conference on Inorganic Membranes, Montpellier, France, 1989, pp. 199-205.

10 F.P. Cuperus, Characterization of ultrafiltration membranes: pore structure and top layer thickness, Ph.D. Thesis, University of Twente, The Netherlands, 1990.

11 F.P. Cuperus, D. Bargeman and C.A. Smolders, Permporometry. 'The determination of the size distribution of active pores in UF membranes, J. Membrane Sci., 71 (1992) 57-67. 
M. Mulder, Basic Principles of Membrane Technol ogy, Kluwer Academic Publishers, Dordrecht, The Netherlands, 1991.

13 S.J. Gregg and K.S.W. Sing, Adsorption, Surface Area and Porosity, 2nd edn., Academic Press, New York, NY, 1982.

14 D. Ruthven, Principles of Adsorption and Desorption Process, John Wiley \& Sons, New York, NY, 1984.

15 K.S.W. Sing, D.H. Everett, R.A.W. Haul, L. Moscou, R.A. Pierotti, J. Rouquerol and T. Siemieniewska, Reporting physisorption data for gas/solid systems with special reference to the determination of surface area and porosity, Pure Appl. Chem., 57(4) (1985) 603-619.

16 Y.S. Lin and A.J. Burggraaf, Preparation and characterization of high-temperature thermally stable alumina composite membranes, J. Am. Ceram. Soc., 74 (1991) 219-224.

17 Y.S. Lin, K.J. de Vries and A.J. Burggraaf, Thermal stability and its improvement of the alumina membranes prepared by sol-gel methods, J. Mater. Sci., 26 (1990) 715-720.

18 A.J. Burggraaf and K. Keizer, Synthesis of inorganic membranes, in: R. Bhave (Ed.), Inorganic Membranes: Synthesis, Characterization and Properties, Van Nostrand Reinhold, New York, NY, 1991, Chap. 2, pp. 10-63.

19 K. Keizer and A.J. Burggraaf, Porous ceramic materials in membrane applications, in: B. Taylor (Ed.), Science of Ceramics, Vol. 14, The Institute of Ceramics, UK, 1988, pp. 83.

20 A. Leenaars, Preparation, structure and separation characteristics of ceramic alumina membranes, $\mathrm{Ph} . \mathrm{D}$. Thesis, University of Twente, The Netherlands, 1984.

21 R.J. Uhlhorn, Ceramic membranes for gas separation: synthesis and transport properties, Ph.D. Thesis, University of Twente, The Netherlands, 1990.

22 G.J.I. Igwe, Powder Technology and Multiphase Systems: Gas Permeametry and Surface Area Measurement, Ellis Horwood, New York, NY, 1991.

23 J.H. de Boer, The shapes of capillaries, in: The Structure and Properties of Porous Materials, D.H. Everett and F.S. Stone (Eds.), Butterworths Scientific Publ., London, 1958, pp. 68-94.

24 E.A. Mason and A.P. Malinouskas, Gas Transport in Porous Media: the Dusty Gas Model, Elsevier, Am sterdam, 1983.

25 R.C. Wilhoit and B.J. Zwolinski, Handbook of Vapor Pressure and Heats of Vaporization of Hydrocarbons and Related Compounds, Texas A \& M University, 1971, pp. 40.

\section{Appendix}

The permporometry measurements were performed under the conditions described previously in the section "Experimental". The overall pressure on both sides of the specimens is about 1 bar and no overall pressure gradient is present. The driving force for the oxygen transport through the specimen is the concentration gradient of the gases supplied on both sides of the specimens. The average pore diameter of the specimens in question is about 20 nm or smaller. Thus the oxygen gas diffusion through the specimens is assumed to be Knudsen diffusion [24]. The Knudsen permeability $F\left(\mathrm{~mol} / \mathrm{m}^{2}\right.$-sec-Pa) through porous media is given by:

$F=\frac{2 \pi v}{3 \tau R T l} \int_{0}^{\infty} r^{3} f(r) \mathrm{d} r$

where $r$ is the pore radius $(\mathrm{m}), \tau$ is the tortuosity, a correction for a deviation from the model pore shape, $l$ the thickness of the porous sample (m), $v$ the average molecular velocity of the permeating gas $(\mathrm{m} / \mathrm{sec}), R$ and $T$ have the common meaning, $f(r)$ is the pore size distribution [the number of pores with radius from $r$ to $r+\mathrm{d} r$ per unit volume $\left(\right.$ in $\left.\mathrm{m}^{-3}\right)$ ].

At the permporometry measurement temperature $\left(15^{\circ} \mathrm{C}\right)$, the gas-solid interfacial tension (of cyclohexane and alumina), $\gamma_{s}$, is $2.352 \times 10^{-2} \mathrm{~J} / \mathrm{m}^{2}$; the molar volume of cyclohexane [25], $\nu$, is $1.079 \times 10^{-4} \mathrm{~m}^{3} / \mathrm{mol}$, the contact angle, $\theta$, is assumed to be zero and thus $\cos \theta=1$, and the process parameter, $n$, is 2 since the desorption process is used [13,14]. Substituting the above data into the Kelvin equation (eqn. 1), the relation between the relative vapour pressure $\left(P_{\mathrm{r}}\right)$ and the Kelvin radius $\left(\rho_{\mathrm{k}} \times 10^{-9} \mathrm{~m}\right)$ is constructed as follows:

$\rho_{\mathrm{k}}=-\frac{2.328}{\ln P_{\mathrm{r}}}$ 
During the permporometry measurement, when the relative vapour pressure of cyclohexane is reduced from unity to $P_{1}$, all pores with Kelvin radius equal to or smaller than $\rho_{1}$ are blocked by capillary condensation according to eqn. (A-2). Thus only those pores with Kelvin radii larger than $\rho_{1}$ are open for oxygen gas diffusion. The subsequent oxygen permeability $\left(F_{1}\right)$ measured is in fact the cumulative value of oxygen permeability, which diffused through pores with Kelvin radii larger than $\rho_{1}$ in the specimen. From eqn. (A-1), the permeability through pores with radii larger than $\rho$ can be calculated according to:

$F(\rho)=\frac{2 \pi v}{3 \tau R T l} \int_{\rho}^{\infty} r^{3} f(r) \mathrm{d} r$

The permeability through all pores with radii between $\rho$ and $\rho+\delta \rho$ is:

$$
\begin{aligned}
& F(\rho+\delta \rho)-F(\rho)= \\
& \frac{2 \pi \mathrm{v}}{3 \tau R T l}\left[\int_{\rho+\delta \rho}^{\infty} r^{3} f(r) \mathrm{d} r-\int_{\rho}^{\infty} r^{3} f(r) \mathrm{d} r\right]= \\
& -\frac{2 \pi v}{3 \tau R T l} \int_{\rho}^{\rho+\delta \rho} r^{3} f(r) \mathrm{d} r
\end{aligned}
$$

As $\delta \rho$ approaches 0 , we have the following relation:

$$
\begin{aligned}
& \lim _{\delta \rho \rightarrow 0}[F(\rho+\delta \rho)-F(\rho)]= \\
& \lim _{\delta \rho \rightarrow 0}-\frac{2 \pi v}{3 \tau R T l} \int_{\rho}^{\rho+\delta \rho} r^{3} f(r) \mathrm{d} r
\end{aligned}
$$

That is:

$\mathrm{d} F=-\frac{2 \pi v}{3 \tau R T l} \rho^{3} f(\rho) \mathrm{d} \rho$

or:

$\frac{\mathrm{d} F}{\mathrm{~d} \rho}=-\frac{2 \pi v}{3 \tau R T l} \rho^{3} f(\rho)$

Under the experimental conditions used, the diffusing gas (oxygen) is assumed to be an ideal gas. Thus the average molecular velocity of the gas is given by:

$v=\sqrt{\frac{8 R T}{\pi M}}$

where $M$ is the molecular weight of the gas $(\mathrm{kg} /$ mol). Substituting eqn. (A-8) into eqn. (A-7) and rearranging it, we have:

$f(\rho)=-\frac{3 \tau l}{2 \rho^{3}} \sqrt{\frac{M R T}{8 \pi}} \frac{\mathrm{d} F}{\mathrm{~d} \rho}$

With this equation, the pore size distribution $f(\rho)$ (dimension $\mathrm{m}^{-3}$ ) can be calculated from the cumulative oxygen permeability, $F$, determined by means of permporometry. 